\title{
Mortalidade infantil "evitável" em duas cidades do Nordeste do Brasil: indicador de qualidade do sistema local de saúde
}

\section{"Avoidable" infant mortality in two cities of Northeastern Brazil: quality indicator of the local health system}

\author{
Zulmira Maria de Araújo Hartz, François Champagne, Maria do Carmo Leal e André- \\ Pierre Contandriopoulos \\ Departamento de Epidemiologia da Escola Nacional de Saúde Pública. Rio de Janeiro, RJ - Brasil \\ (Z.M.A.H., M.C.L.), Groupe de Recherche Interdisciplinaire en Santé. Faculté de Médicine de \\ I'Université de Montreal. Canada (F.C., A.P.C.)
}

\begin{abstract}
Resumo
Objetiva-se conhecer a magnitude e a estrutura de causalidade da mortalidade infantil - considerando-a um "evento sentinela" da qualidade da assistência à saúde - em dois municípios do Nordeste, Brasil. Trata-se de estudo de base populacional, do tipo "experimentação invocada", que compara a mortalidade infantil observada com aquela esperada, dado um programa de atenção à saúde materno-infantil operando a contento, permitindo calcular um "índice de mortes evitáveis" (PDI). Realizaram-se uma busca ativa e uma investigação epidemiológica dos óbitos, visando a eliminar o sub-registro desses eventos. As taxas de mortalidade infantil, embora relativamente baixas - 39 e 44 por 1.000 nascidos vivos, respectivamente - correspondem a um PDI de $40 \%$, o que significa uma estrutura de causalidade compatível com taxas de mortalidade de 100 por 1.000 nascidos vivos. Esses achados sugerem uma distribuição desigual dos óbitos, confirmada por uma análise comparativa entre a população de baixa renda e outras categorias de renda (com razões de risco de 8 e 17,6 para a mortalidade infantil total e para a mortalidade infantil por doenças infecciosas, respectivamente). O PDI mostrou-se válido enquanto um índice de evitabilidade dos óbitos infantis, com a vantagem de poder ser utilizado de forma simples e fácil por gerentes de sistemas de saúde preocupados com a qualidade dos programas voltados para mães e filhos.
\end{abstract}

Mortalidade infantil. Evento sentinela. Qualidade dos cuidados de saúde.

\begin{abstract}
This paper seeks to discover the magnitude and causality structure of infant mortality - considered a "sentinel event" for quality-of-care indexes in health - in two municipalities of Northeastern Brazil. This is a population based study of the "invoked experimentation" type comparing observed infant mortality with that expected, given a properly functioning maternal and infant care program, allowing for the calculation of a "preventable index" (PDI) for
\end{abstract}

Correspondência para/Correspondence to: Zulmira Maria de Araújo Hartz - Departamento de Epidemiologia da Escola Nacional de Saúde Pública. R. Leopoldo Bulhões, 1480 - $8^{\circ}$ andar - 21041-210 Rio de Janeiro, RJ - Brasil.

Recebido em 27.10.1994. Reapresentado em 26.6.1995. Aprovado em 6.11.1995. 
these two municipalities. The preliminary step consisted of an active search and epidemiological investigation of deaths in order to eliminate their underreporting as events. Infant mortality rates in the two areas were relatively low - 39 and 44 per thousand live births, respectively -, but PDI in both was classified of the order of $40 \%$, thus indicating a causality structure compatible with mortality rates of 100 per thousand. These findings suggest an uneven distribution of deaths, proved by an analytical comparison of the low income population with that of other income brackets (with risk ratios of 8 and 17.6 for total infant mortality and infant mortality from infectious diseases, respectively). PDI proved to be a useful index of preventability of infant deaths, and has the advantage of being simple and easy for health system managers concerned with the quality of health programs devoted to mothers and children to use.

Infant mortality, Preventable deaths. Sentinel event. Quality of health care.

\section{INTRODUÇÃO}

A mortalidade infantil como ocorrência "evitável" por serviços de saúde eficazes é conhecida desde os anos 70, constituindo-se, portanto, um "evento sentinela" da qualidade da atenção médica (Rutstein e col. $\left.{ }^{41}, 1976\right)$. Em países onde o risco de morrer dos menores de 1 ano permanece elevado, a necessidade de se obter estimadores de qualidade que evidenciem esta problemática não é apenas uma exigência metodológica, mas ética, por que implica a "mortalidade consentida" de crianças.

No Brasil, onde o sub-registro médio de óbitos em geral é de $25 \%$ (Becker e col. ${ }^{4}, 1989$ ) e as causas "maldefinidas" constituem, na região Nordeste, até 45\% dos óbitos registrados (Araújo ${ }^{2}, 1992$ ), a magnitude da mortalidade infantil tem sido aproximada por estimadores demográficos de sub-registros internacionalmente reconhecidos $\left(\mathrm{Blom}^{8}, 1987\right.$; Ferreira e Flores $^{19}$, 1990; Simões ${ }^{46}$, 1989; Szwarcwald e col. ${ }^{48}$, 1992; UNICEF ${ }^{50}, 1990$; UNICEF $^{52}$, 1991). Um problema constatado são as divergências de informações geradas pelos diferentes métodos, que, por exemplo, estimam a queda da mortalidade infantil para o País, entre 1980 e 1987, de 82 a 66 por 1.000 (Szwarcwald e $\operatorname{col}^{48}, 1992$ ) e de 75 a 51 por 1.000 em outro estudo (UNICEF ${ }^{50}, 1990$ ). Se o consenso sobre a tendência de declínio existe, a questão das desigualdades continua fazendo com que, no Nordeste do País, onde as taxas são as mais elevadas, os valores, para o mesmo período, passem de 116 a 107 por 1.000 , no primeiro estudo, e de 115,7 a 76,6 por 1.000 , no segundo.

Os estimadores têm também se mostrado insuficientes para a reorientação das ações em nível local, modelo assistencial preconizado pelo Sistema Único de Saúde (SUS). Eles se baseiam em amostras para grandes regiões ou capitais, sem considerar os diferenciais de sub-registro dos municípios menores, e muitos omitem o perfil de causalidade (Hartz e col. ${ }^{24}, 1996$; $\mathrm{Saad}^{43}$, 1983). Como lembram Nakamura e col. ${ }^{30}$ (1991): "subestimar a mortalidade infantil pode ter efeitos danosos. Problemas de financiamento dos programas de saúde materno-infantil poderão ocorrer em virtude da menor prioridade dada às questões da saúde infantil (...) O exame das causas de óbito tem sido importante no planejamento de estratégias visando a reduzir a mortalidade infantil (...) Dados locais são também necessários para conscientizar a população e dar apoio a programas de intervenção".

O desconhecimento de informações compromete também a validade da medida, pois se há muitos resultados desconhecidos, os achados não poderão mostrar a situação real (Abramson ${ }^{1}$, 1988; Rosemberg $\left.^{39}, 40,1989\right)$.

As lacunas de conhecimento desta problemática são evidentes e os estudos nacionais têm concentrado sua atenção sobre os determinantes socioeconômicos da mortalidade infantil (Becker ${ }^{5}$, 1988; Paim e col..$^{32}$, 1987; Sawyer e col..$^{44}$, 1987; Simões e Ortiz ${ }^{45}$, 1988; Szwarcwald e col. ${ }^{49}, 1992$ ), de indiscutível importância, pouco se cobrando dos sistemas de saúde, não obstante a eficácia das ações dirigidas a este grupo ter sido comprovada em diferentes contextos e, mesmo, em populações de baixa renda (Goulet ${ }^{22}$, 1985; Nersesian ${ }^{31}$, 1988; UNICEF $^{51}$, 1986; Wash e Warren $^{54}, 1980$ ), permitindo-nos categorizá-los como "óbitos evitáveis".

O conceito de óbitos evitáveis, ou sentinel events, foi proposto por Rutstein e col. ${ }^{41}$ (1976), considerando que estas condições poderiam ser melhoradas por ações de saúde eficazes, consituindo-se clearcut, índices da qualidade da assistência à saúde de 
uso imediato. Vários autores têm proposto estes indicadores para avaliação da eficácia de sistemas de saúde (Charlton e col. ${ }^{13}$, 1983; Duchiade e col. ${ }^{17}$, 1989; Guimarães e Fischman ${ }^{23}$, 1985; Jougla e col. ${ }^{25}$, 1987; López ${ }^{27}$, 1989), uma forma de "monitoramento de emergência" (Rutstein ${ }^{42}$, 1987).

Diversos autores têm opinado sobre as vantagens e desvantagens do método, a maioria enfocando os óbitos evitáveis, e algumas considerações são importantes de ressaltar. Para Murnaghan ${ }^{29}$ (1981), a mortalidade infantil seria o primeiro destes indicadores, nos países em desenvolvimento, a ser claramente relacionado ao estado de saúde de uma população e à qualidade dos serviços. Plaut e Roberts ${ }^{34}$ (1989) lembram que os "eventos" escolhidos podem ser apenas indicadores da qualidade de serviços (outcome measures) e não da saúde de uma população, como a mortalidade perinatal nos países desenvolvidos. Charlton ${ }^{12}$ (1987) sublinha que a mortalidade é também função da incidência do problema, mas que isto deve ser argumento para que a rede de serviços amplie sua cobertura nas àreas de maior incidência, e, se o condicionamento socioeconômico dos problemas de saúde é uma realidade, é responsabilidade dos sistemas de saúde serem mais accessíveis e eficazes onde os riscos são mais elevados pela precariedade do meio ambiente. Este tipo de análise, semelhante àquele que tem sido feito por alguns autores brasileiros (Guimarães e Fischman ${ }^{23}$, 1985; Monteiro $^{28}$, 1986; Paim e col..$^{32}$, 1987; Yunes e Campos $^{55}$, 1989), deve ser também uma atribuição dos serviços, como ressaltam Evans e col. ${ }^{18}$ (1981), lembrando que cabe ao sistema de saúde demonstrar a importância do engajamento político para reduzir essas desigualdades.

Assim, a desigualdade do risco de ocorrência dos problemas de saúde é também uma medida da evitabilidade dos eventos e, conseqüentemente, da qualidade dos sistemas de saúde. A multiplicidade de opiniões na abordagem da "evitabilidade" da mortalidade infantil evidencia sua força como o mais sensível dos indicadores propostos para evento sentinela da qualidade dos serviços, levando-se em conta a possibilidade de melhores dados e a concentração de investimentos públicos nesta área (Paim e col. ${ }^{32}$, 1987, Buck e Bull ${ }^{9}$, 1986).

O objetivo do presente trabalho é construir o perfil do que seria a "mortalidade infantil evitável", partindo da busca ativa e da investigação epidemiológica dos óbitos de menores de 1 ano em dois municípios do Nordeste (Açu e Caicó - SILOS* 1 e
SILOS 2), cuja população alvo é estimada em 1.069 e 1.226 nascidos vivos, respectivamente. A pesquisa insere-se num conjunto de investigações integradas sob a forma de "estudos de caso" desses SILOS, que têm como objetivo geral fornecer subsídios a uma melhoria da eficácia das ações do Programa Materno-Infantil na redução da mortalidade dos menores de 1 ano em toda região.

\section{METODOLOGIA}

Para estimar a mortalidade infantil considerada evitável foi utilizada uma estratégia de pesquisa do tipo experimentação invocada, proposta por Contandriopoulos e col. ${ }^{14}$ (1989), em que a mortalidade infantil observada é comparada à mortalidade infantil esperada (população padrão), se o Programa Materno-Infantil (PMI) funcionasse adequadamente nos SILOS. A validade interna desta estratégia de avaliação é dada pela própria validade dos estudos que deram suporte à categorização dos eventos sentinela por Rutstein e col. ${ }^{41}$ (1976).

Os indicadores da mortalidade observada e esperada (menor risco) foram obtidos através dos coeficientes de mortalidade infantil dos diversos estratos populacionais, por grupo etário (mortalidade neonatal, correspondendo aos menores de 28 dias, e mortalidade pós-neonatal, aos demais menores de 1 ano), e pela estimativa do Preventable Deaths Index (PDI), proposta por D'Sousa ${ }^{15}$ (1989).

A distribuição dos óbitos por estratos populacionais procura evidenciar as desigualdades entre áreas com diferenciais de risco e utiliza a causa básica e causas associadas na análise da mortalidade proporcional. Os intervalos de confiança $(95 \% \mathrm{CI})$ para as estimativas do risco relativo (RR) são calculados com base no erro padrão da razão de dois coeficientes independentes (Dever e Champagne ${ }^{16}$, 1984; Kleinman ${ }^{26}$, 1977).

A inclusão do PDI permite sumarizar melhor os dados e redimensionar as informações. Esse índice foi construído com a perspectiva dos países em desenvolvimento, a partir da análise de uma experiência em Bangladesh para a redução da mortalidade infantil (19661982), incluindo diferentes momentos de transição epidemiológica, e sua utilidade de aplicação já foi testada na Ásia e na África. Baseando-se numa transformação estatística das medidas demográficas usuais (logarithm of the odds of dying), o índice estabelece uma correlação entre os níveis de mortalidade e a estrutura das causas dos óbitos. O PDI também integra a noção de dificuldade de controle (custo/factibilidade) dos trabalhos de Wash e Warren ${ }^{54}$ (1980) e se apresenta sob a forma simples de uma escala percentual, facilitando sua interpretação. Seu valor é calculado pelo diferencial dos Índices de Mortalidade Infantil (IMI) da população de estudo e da população padrão. Quando as causas dos óbitos e a estimativa dos custos

* SILOS - Sistema Local de Saúde 
encontram-se disponíveis, usa-se a fórmula PDI = IMI. $\sum \mathrm{i}$ (Mi/M.Cfi).

$$
\begin{aligned}
\mathrm{Mi}= & \text { coeficiente de mortalidade pela causa } i \\
\mathrm{M}= & \text { coeficiente de mortalidade por todas as cau- } \\
& \text { sas } \\
\mathrm{Cfi}= & \text { fator custo-factibilidade (valores entre } 0-1) \\
& \text { para prevenir óbitos pela causa } i \\
\mathrm{IMI}= & 100-\mathrm{A}\left[\ln \left\{1^{\mathrm{q}} 0 / 1-\left(1^{\mathrm{q}} 0\right)+\mathrm{B}\right\}\right]
\end{aligned}
$$

A taxa de mortalidade infantil (TMI) é convertida em escala variando de $0(\mathrm{TMI}=5 / \%$ o) a $100(\mathrm{TMI}=300 / \%$ o), ou Índice de Mortalidade Infantil. A e B são constantes e uma tabela com os valores de IMI correspondentes à TMI observada facilita o cálculo do PDI (Anexo).

Assume-se que IMI = 30 reflete a "população padrão", este valor sendo tomado como indicação da eliminação da maioria das causas preveníveis de origem infecciosa.

Para estimar o Cfi (Tabela 1), foram aplicados os critérios de "redutibilidade por causa de morte" (9르 Revisão da CID) da Fundação SEADE²1 (1991), introduzindo a seguinte ponderação para os óbitos ocorridos:

$$
\begin{array}{ll}
\text { Cfi }=1: & \begin{array}{l}
\text { reduzíveis por prevenção ou diagnóstico } \\
\text { e tratamento precoces; }
\end{array} \\
\text { Cfi }=0,8: & \begin{array}{l}
\text { reduzíveis por adequada atenção à gravi- } \\
\text { dez e ao parto; }
\end{array} \\
\text { Cfi }=0,6: & \text { parcialmente reduzíveis; } \\
\mathrm{Cfi}=0,5: & \text { causas maldefinidas e outras causas; } \\
\mathrm{Cfi}=0: & \text { causas não evitáveis }
\end{array}
$$

O tratamento dos dados foi feito com Epi Info 5.1.

\section{Validando a Medida da Mortalidade Infantil}

A validade de uma medida refere-se à sua adequação ao que poderíamos chamar "significados comumente aceitos de um determinado conceito", de maneira que os julgamentos feitos pareçam razoáveis $\left(\mathrm{Babbie}^{3}, 1986\right.$; Streiner e Norman ${ }^{47}, 1989$ ).

Uma advertência de $\mathrm{Babbie}^{3}$ (1986) é de que qualquer medida muito confiável e específica reduz a riqueza da significação conceitual, e a melhor solução para aumentar sua validade de conteúdo "é utilizar diferentes medidas que abordem diferentes aspectos do conceito".
Potvin $^{36}$ (1984) observa que a validade da informação dos óbitos aumenta com a revisão de prontuários e a utilização de somente três dígitos da Classificação Internacional de Doenças (CID). Observação semelhante foi feita por Carvalho e col. ${ }^{11}$ (1990), cuja investigação permitiu reduzir em $50 \%$ as causas genéricas ou maldefinidas.

Puffer $^{37}$ (1989) defende o estudo da multicausalidade (causa básica e associadas), o que parece indispensável à melhoria da "validade de conteúdo", como já foi evidenciado na Investigação Interamericana de Mortalidade na Infância (Puffer e Serrano ${ }^{38}$, 1973). Nesse estudo pôde-se constatar a magnitude de problemas como a desnutrição, que, por exemplo, só aparecia como causa básica em 1,5\% dos óbitos do Recife (cidade do Nordeste do Brasil), mas estava presente em $34 \%$ dos óbitos dos menores de 1 ano.

No presente estudo, inicialmente foi feito um levantamento dos óbitos de menores de 1 ano, ocorridos nos 12 meses que antecederam a pesquisa (setembro de 1990 a agosto de 1991), no registro dos cartórios, nas unidades hospitalares e na relação de sepultamentos de igrejas e cemitérios locais ("busca ativa"), distribuindo-se todas as ocorrências por setor censitário. Os atestados de óbito foram codificados por um profissional treinado pelo Centro da Organização Mundial da Saúde para Classificação de Doenças em Português (São Paulo).

A coleta dos dados foi programada para ocorrer na mesma época do último recenseamento, de modo a obter a melhor aproximação possível do número de nascidos vivos no período e, assim, dos estimadores de mortalidade infantil para o total dos municípios, setores urbanos e rurais.

Foram efetuadas entrevistas domiciliares com os familiares para identificar sua(s) causa(s) e indagar sobre os motivos da não-utilização dos serviços de saúde. A entrevista visava também complementar informações das Declarações de Óbito ligadas à mãe (idade, instrução, número de filhos, tipo de parto) ou à criança (peso ao nascer) e obter outras julgadas de interesse para a compreensão da ocorrência: história gestacional, complicações do parto, amamentação, imunizações e outros problemas de saúde da criança.

O questionário utilizado é uma adaptação do instrumento elaborado por Puffer e Serrano ${ }^{38}$ (1973), vali-

Tabela 1 - Distribuição proporcional de óbitos de menores de 1 ano por causa básica* segundo critérios de redutibilidade ponderados por custo-factibilidade (CF) em dois SILOS do Nordeste do Brasil. De setembro 1990 a agosto 1991.

\begin{tabular}{lcc}
\hline Critérios & $\begin{array}{c}\text { SILOS**1 } \\
\text { Cfi (Mi/M) } \\
\left(\mathrm{N}^{\circ}=42\right)\end{array}$ & $\begin{array}{c}\text { SILOS** } 2 \\
\text { Cfi }(\mathrm{Mi} / \mathrm{M}) \\
\left(\mathrm{N}^{\circ}=54\right)\end{array}$ \\
\hline Reduzíveis por prevenção, diagnóstico ou tratamento precoce & 0,64 \\
(009, 482, 486, 560) & 0,06 & 0,54 \\
Reduzíveis por adequada atenção à gravidez ou parto (762, 768, 771) & - & 0,03 \\
Parcialmente reduzíveis (765, 769) & 0,13 & 0,08 \\
Maldefinidas e outras causas (271, 770, 772, 776, 779, 798) & 0,10 \\
Não evitáveis (740, 741, 746) & 0,83 & 0 \\
\hline Total & 0,75 \\
\hline
\end{tabular}

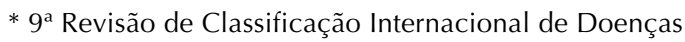

** SILOS - Sistema Local de Saúde 
dado e aplicado em estudo realizado no Rio de Janeiro (Carvalho e col. ${ }^{11}$, 1990; Duchiade e col. ${ }^{17}$, 1989). Para reduzir os problemas ligados à memorização dos fatos pelos entrevistados — recall of health events (Cannel e col. $\left.{ }^{10}, 1977\right)$ - trabalhou-se com um calendário, destacando as festas populares desses municípios, e solicitou-se que as questões fossem respondidas por duas pessoas envolvidas com a criança. As entrevistas foram realizadas por uma enfermeira ligada à equipe da pesquisa, sendo acompanhada por uma auxiliar do nível local para permitir a reutilização do instrumento de forma continuada em investigações futuras, conforme interesse dos próprios coordenadores do PMI das áreas investigadas.

Uma revisão dos prontuários dos óbitos hospitalares foi feita por um pediatra "externo", e o coeficiente kappa de concordância (Fleiss ${ }^{20}, 1981$ ) foi estabelecido para analisar a confiabilidade da informação da causa básica (grandes grupos da CID). Estes procedimentos foram feitos somando-se os óbitos dos dois municípios cujos prontuários foram localizados (Puffer e Serrano ${ }^{38}$, 1973), o que representou $80 \%$ do total. A decisão da análise conjunta, aumentando o tamanho da amostra, foi coerente com o pressuposto de que o preenchimento de atestados remete a problemas de educação médica, e não às peculiaridades dos SILOS.

\section{RESULTADOS}

\section{Elementos para Crítica dos Dados}

Os coeficientes kappa de concordância usados como medidas de confiabilidade para os grandes grupos da CID mostraram os seguintes valores: grupo I (doenças infecciosas) $=30$; grupo VIII (doenças do aparelho respiratório) $=65$; e grupo XV (causas perinatais $)=51$.

Apesar de o coeficiente kappa ser uma medida conservadora dos acordos verdadeiros (Bernard e Lapointe $^{7}$, 1987) e os resultados serem semelhantes aos de Carvalho e col. ${ }^{11}$ (1990), preocupou o baixo valor observado nas doenças infecciosas (IC de 1 a 59), ratificando a importância da busca ativa para a redução dos viéses da medida. Considerando-se o observador externo como gold standard, verificouse, neste grupo, baixa sensibilidade $(33,3 \%)$ e alta especificidade $(93,3 \%)$. Aliás, poderia mesmo chamar de "grupo das diarréias" — nenhum caso de doença imunizável ou de doenças infecciosas está contemplado no grupo - , alertando para a ocorrência precoce deste problema, já estimado como causa

Tabela 2 - Perfil da mortalidade infantil antes e depois da busca ativa de óbitos em dois SILOS do Nordeste do Brasil, setembro 1990 a agosto 1991.

\begin{tabular}{|c|c|c|c|c|c|c|}
\hline & \multicolumn{3}{|c|}{ SILOS** 1} & \multicolumn{3}{|c|}{ SILOS** 2} \\
\hline & Antes & Depois & $\mathrm{D} / \mathrm{A}$ & Antes & Depois & $\mathrm{D} / \mathrm{A}$ \\
\hline \multicolumn{7}{|l|}{ Indicadores } \\
\hline Mortalidade infantil* & 14 & 39 & 2,8 & 34 & 44 & 1,3 \\
\hline Mortalidade neonatal* & 9 & 13 & 1,4 & 15 & 23 & 1,5 \\
\hline Mortalidade pós-neonatal* & 5 & 26 & 5,2 & 19 & 21 & 1,1 \\
\hline Mortalidade infantil urbana* & 14 & 40 & 2,9 & 35 & 47 & 1,3 \\
\hline Mortalidade infantil rural* & 5 & 38 & 7,6 & 25 & 30 & 1,2 \\
\hline \multicolumn{7}{|l|}{ Mortalidade proporcional (\%) } \\
\hline I Doenças infecciosas (009 - 139) & 6,7 & 42,9 & 6,4 & 7,1 & 42,6 & 6,0 \\
\hline $\begin{array}{l}\text { III Doenças de causas nutricionais e } \\
\text { endócrinas }(240-279)\end{array}$ & - & 2,4 & 2,4 & - & - & - \\
\hline VIII Aparelho respiratório (460 - 519) & 26,7 & 31,4 & 0,8 & 4,8 & 7,4 & 1,5 \\
\hline IX Aparelho digestivo (520 - 579) & - & - & - & 2,4 & 3,7 & 1,5 \\
\hline XIV Anomalias congênitas (740 - 759) & 6,7 & 2,4 & 0,4 & 4,8 & 5,6 & 1,2 \\
\hline XV Causas perinatais $(760-779)$ & 53,3 & 23,8 & 0,4 & 40,5 & 35,2 & 0,9 \\
\hline XVI Causas maldefinidas (780 - 799) & 6,7 & 7,1 & 1,1 & 40,5 & 5,6 & 0,1 \\
\hline \multicolumn{7}{|l|}{ Causas associadas ( $\%)$} \\
\hline Prematuridade & 6,7 & 12,2 & 1,8 & 9,5 & 12,2 & 1,3 \\
\hline Desnutrição & 13,3 & 34,7 & 2,6 & 4,8 & 24,5 & 5,1 \\
\hline Diarréia/desidratação & - & 8,2 & 8,2 & - & 6,1 & 6,1 \\
\hline Septicemia & - & 4,1 & 4,1 & 4,8 & 6,1 & 1,3 \\
\hline Infecções respiratórias & - & 18,4 & 18,4 & - & 10,2 & 10,2 \\
\hline Outras & 13,3 & 4,1 & 0,3 & - & - & - \\
\hline
\end{tabular}

* Coeficientes por 1.000 nascidos vivos

** SILOS: Sistema Local de Saúde

D/A: Depois/Antes 
básica de $32 \%$ dos óbitos neonatais em estudo recente (Bennet $\left.{ }^{6}, 1990\right)$.

Outros indicadores da baixa qualidade da informação na Declaração de Óbito são a proporção de causas maldefinidas e o baixo registro das causas intercorrentes, minimizados após a busca ativa (Tabela 2). As causas maldefinidas são, na verdade, codificações para óbitos sem "assistência médica". Ao se incluir os diagnósticos "prováveis" de todas as crianças que receberam assistência médica nas últimas 48 horas que antecederam os óbitos de ocorrência domiciliar, o valor preditivo positivo destas declarações é de apenas $28 \%$, o que mostra, também, que "sem assistência médica" significaria falta de resolutividade, e não ausência da assistência.

A importância da desnutrição entre as causas associadas (chegando a 35\% no SILOS 1 após a busca ativa), mas inexistente entre as causas principais, confirma, mais uma vez, a imagem de "desnutrição invisível" na vida e morte destas crianças (Carvalho e col. ${ }^{11}$, 1990; Puffer e Serrano ${ }^{38}$, 1973; Wallace ${ }^{53}$, 1990).

A busca ativa, como esperado, ampliou a magnitude da mortalidade infantil e introduziu modificações importantes na sua composição, com o predomínio da mortalidade pós-neonatal e incremento elevado da mortalidade rural no SILOS 1. Já no SILOS 2 chama a atenção a mortalidade neonatal, que supera a pós-neonatal (Tabela 2).

\section{A Mortalidade Evitável}

Se fossem utilizados apenas os diferenciais da mortalidade infantil para calcular o PDI, vê-se que os coeficientes de mortalidade infantil no SILOS 1 (39;IC 27.2-50.8) e SILOS 2 (44; IC 32.2-55.8) não são significativamente diferentes, com PDI $=20 \%$ nos dois SILOS, o que corresponderia a um nível intermediário no perfil de mortalidade entre as cau- sas ditas "endógenas" (hard rock) e "exógenas" (soft rock). Estas últimas são aquelas que "poderiam ser controladas através de medidas de saúde pública, imunização e antibióticos".

Estimando o cálculo do PDI pela fórmula apresentada na metodologia, em que efetua-se o produto do IMI (verificado no Anexo) pelos valores da Tabela 1 (SILOS $1=0,83$ e SILOS $2=0,75)$, teria, aproximadamente, $\mathrm{um} P D I=40 \%$. Isto significaria dizer que o perfil de causalidade nos dois municípios é condizente com uma taxa de mortalidade infantil de 100 por 1.000 nascidos vivos, estando ambos na faixa de soft rock, ou seja, grande parte dos óbitos podendo ser evitados pelas ações do Programa Materno-Infantil.

Este comportamento é próprio das grandes desigualdades de risco no interior dos SILOS, o que foi possível verificar no SILOS 2 analisando-se uma concentração populacional bem-delimitada (8.093 habitantes), de baixa renda (BR), o que permitiu, pela coincidência com um agregado de setores censitários, estudar os diferenciais de risco entre grupos socioeconômicos distintos, cujo PDI varia de 50 (BR) a 0 (outros), com a razão de riscos variando de 5,9 a 17,6 vezes (Tabela 3).

Salienta-se a coerência com um estudo realizado em Porto Alegre (Guimarães e Fischmann ${ }^{23}$, 1985), que apresentou uma taxa de mortalidade infantil de 35 por 1.000 , mas também com diferenças de 160 a 12 por 1.000 nascidos vivos entre comunidades de baixa renda e outras.

\section{DISCUSSÃO}

Os coeficientes de mortalidade infantil são indicadores privilegiados, possibilitando a comparação dos diferentes grupos populacionais, mas os problemas de qualidade concernentes à sua medida não podem ser negligenciados (Rosemberg $\left.{ }^{40}, 1989\right)$. A

Tabela 3 - Perfil da mortalidade infantil (por 1.000 nascidos vicos) em uma área urbana de baixa renda e nos outros setores urbanos do SILOS* 2 .

\begin{tabular}{lccc}
\hline Indicadores & Baixa renda $\left(\mathrm{N}^{\circ}=202\right)$ & Outros $\left(\mathrm{N}^{\circ}=1.024\right)$ & $\mathrm{RR}^{* * *}$ \\
\hline Mortalidade infantil & 160 & 20 & 8,0 \\
Mortalidade neonatal** & 93 & 10 & 9,3 \\
precoce & 0,6 & 0,9 & \\
tardia & 0,4 & 0,1 & 6,7 \\
Mortalidade pós-neonatal & 67 & 10 & 17,6 \\
Mortalidade por doenças infecciosas & 88 & 5 & 5,9 \\
Mortalidade por causas perinatais & 47 & 8 & \\
\hline
\end{tabular}

* SILOS: Sistema Local de Saúde

** Neonatal precoce: óbitos $<7$ dias

Neonatal tardio: óbitos de 7 a 28 dias

*** Risk ratios $(\mathrm{p}<0.05)$ 
literatura apresentada leva a concluir que a mortalidade infantil evitável pode ser apreendida por medidas diversas de suscetibilidade dos problemas de saúde às intervenções e pelos "excessos" de óbitos em estratos populacionais. Embora conscientes de que nenhum tipo de coleta de dados é isento de vícios ou dificuldades na sua utilização (Potvin e Champagne ${ }^{35}$, 1986), a presente investigação preocupou-se em responder a estas duas questões:

- Os dados do Índice de Mortes evitáveis (PDI) evidenciam a evitabilidade dos óbitos?

- As taxas de pequenas áreas são estáveis o suficiente para refletir os diferenciais de risco a que estão submetidas estas populações?

O PDI, como qualquer índice, ocasionou uma perda de detalhes nas informações, mas, no âmbito geral, a utilização de outros indicadores desagrega a informação, e o PDI traz outras vantagens. Assim, os pequenos sítios de observação, sem base de dados confiável, beneficiam-se com a estabilidade da "população padrão", construída levando-se em conta as séries históricas das patologias mais comuns na casuística dos óbitos. D'Souza ${ }^{15}$ (1989)vê outras vantagens no estudo de sistemas locais de saúde: "Estudos de pequenas áreas podem ser utilizados para validar estimativas indiretas da causa da estrutura dos óbitos (...) fundamentais para uma compreensão mais precisa acerca do nível de mortalidade".

No presente caso ficou clara a necessidade de se conhecer o perfil de causalidade para evitar a subestimação da proporção de ocorrências preveníveis, e a ponderação dos critérios de redutibilidade, como aproximação do Cfi , mostrou-se coerente e relevante para fortalecer a validade de construção da medida. Assumir a omissão de "custos" detalhados decorreu não só das dificuldades de se obter esses valores, mas também do reconhecimento de que já se ultrapassou o equivalente a US\$1.0/habitante/ano, custo suficiente para cobrir as ações do PMI (UNICEF $\left.{ }^{51}, 1986\right)$, buscando-se agora indicadores de qualidade que possam "monitorar" e reorientar estas ações.

Se a tendência decrescente da mortalidade infantil, mesmo em momentos de agravamento da crise socioeconômica, é indicativa da importância das ações de saúde, tais como controle das doenças imunizáveis, ausentes em nosso obituário, por outro lado, a persistência das causas evitáveis e de altas taxas de risco, nos grupos menos favorecidos comprova a localização da problemática, antes comunitária, em determinadas categorias sociais e a omissão dos serviços de saúde na sua resolução. Não se deve esquecer que o conceito de "evento sentinela" obriga a investigação de cada ocorrência tida como evitável no nível individual ou coletivo, propondose as medidas pertinentes. A sua aplicação à mortalidade infantil não pode se restringir à atenção individual, uma vez que as intervenções organizam-se em bases populacionais, constituindo "uma necessidade aguda de monitorar vários grupos populacionais, a fim de identificar uma deterioração súbita no estado de saúde provocada por fatores encômicos e políticos" (Guimarães e Fischmann ${ }^{23}$, 1985).

A presença dos serviços de saúde, de custo compatível com ações potencialmente eficazes, não está impedindo que permaneçam taxas de mortalidade similares às dos Estados Unidos do início do século, e os programas têm que enfrentar o problema, como nas experiências que fundamentaram o modelo dos eventos sentinelas, eliminando as diferenças in a differential fashion (Pharoah e Morris ${ }^{33}$, 1979).

Sem pretender afirmar uma relação de causa-efeito, passível de se enquadrar numa falácia ecológica, é necessário levantar a hipótese de que haja mesmo problemas de "iatrogenia", quando se observa uma taxa de mortalidade neonatal superior à pós-neonatal (SILOS 2), chegando a 93 por 1.000 (BR), e onde a cesariana foi praticada em $54 \%$ dos nascimentos dessas crianças. Esses dados preocupantes estão distantes dos $15 \%$ que se pretende atingir no ano 2000, em nível internacional (Zahniser e col. $\left.{ }^{56}, 1992\right)$, utilizando-se, inclusive, estratégia de investigação caso-acaso, similar à do evento sentinela, já testada anteriormente.

É pretensioso querer, de maneira exaustiva, argumentar sobre a validade das informações obtidas para a operacionalização do conceito de mortalidade infantil evitável. Se este modelo pode ser criticado, é provavelmente por seu caráter "conservador", tolerando como mortalidade infantil "não evitável" uma taxa de $20 \%$ o (PDI = 0). Isto aumenta, no entanto, a especificidade do indicador, condição desejável quando se trabalha com sistemas de informação precários, como é o caso dos países em desenvolvimento. Pode-se, portanto, acrescentar nas conclusões que o PDI representa um estimador da proporção mínima dos óbitos que poderiam ser evitados sem custos financeiros adicionais.

Quanto à validade social do presente estudo, sua evidência dar-se-á no momento que a "revelação dos dados" se transformar em "dados da revolução" da sobrevivência infantil para a qual tem-se as armas; é só lutar... 


\section{REFERÊNCIAS BIBLIOGRÁFICAS}

1. ABRAMSON, J. H. Making sense of data. New York, University Press, 1988.

2. ARAÚJO, J. D. Polarização epidemiológica no Brasil. Inf. Epidemiol. SUS, 1 (2): 5-16, 1992.

3. BABBIE, E. The practice of social research. Belmont, CA, Wadsworth Publ., 1986.

4. BECKER, R. A.; LIMA, D. D.; LIMA, J. T. F.; COSTA JR., M. L. Investigação sobre perfis de saúde, Brasil, 1984. Brasília, Ministério da Saúde, 1989.

5. BECKER, R. A. Brasil: principais causas de mortalidade infantil. In: Crise e infância no Brasil: o impacto das políticas de ajustamento econômico. São Paulo, IPE/USP, 1988. p. 269-80.

6. BENNET, F. J. Child survival. In: Wallace, H. M. \& Giri, K., ed. Health care of women and children in developing countries. California, Third Party Publ. Company, 1990. p. 364-81.

7. BERNARD, P. M. \& LAPOINTE, C. Mesures statistics in epidemiologie. Québec, Presses de I'Université du Québec, 1987.

8. BLOM, D. J. H. Retrospective determination of a child survival ratio suited to Third World situations. In: Measurement in health promotion and protection. Copenhagen, WHO/EA, 1987. p. 565-9.

9. BUCK, C. \& BULL, S. Preventable causes of death versus infant mortality as an indicator of the quality of health services. Int. J. Health Serv., 16: 553-63, 1986.

10. CANNEL, C. F.; MARQUIS, K. H.; LAURENT, A. A summary of sudies of Interviewing methodology. Vital Health Stat. Series 2 (69) 1997.

11. CARVALHO, M. L.; NIOBEY, F. M .L.; MIRANDA, N. N.; SABROZA, P. C. Concordância na determinação da causa básica e óbito em menores de um ano na região metropolitana do Rio de Janeiro. Rev. Saúde Pública, 24: 20-7, 1986.

12. CHARLTON, J. R. H. Avoidable deaths and diseases as monitor of health promotion. In: Measurement in health promotion and protection. Copenhagen WHO/IEA, 1987. p. 467-79.

13. CHARLTON, J. R. H.; SILVER, R.; HARTLEY, R. M.; HOLLAND, W. W. Geographical variation in mortality from conditions amenable to medical interventions in England and Wales. Lancet, 3: 691-6, 1983.

14. CONTANDRIOPOULOS, A. -P.; CHAMPAGNE, F.; POTVIN, B. L.; DENIS, J. -L.; BOYLE, P. Savoir preparer une recherche. Montréal, GRIS Univ. de Montréal, 1989.

15. D'SOUZA, S. The assessment of preventable infant and child deaths in developing countries: some applications of a new index. Rap. Trim. Statist. Sanit. Mond., 42: 16-25, 1989.

16. DEVER, G. E. A. \& CHAMPAGNE, F. Epidemiology in health services management. Rockville, Aspen, 1984.

17. DUCHIADE, M. P.; CARVALHO, M. L.; LEAL, M. C. As mortes em domícilio de menores de um ano na região metropolitana do Rio de Janeiro em 1986: um evento sentinela na avaliação dos serviços de saúde. Cad. Saúde Pública, 5: 251-63, 1989.
18. EVANS, J. R.; HALL, K. L. WARFORD, J. Health care in developing world: problems of scarcity and choice. New Engl. J. Med., 305: 1117-27, 1981.

19. FERREIRA, C. E. C. \& FLORES, L. P. O. Informações sobre a mortalidade infantil para o monitoramento de programas de saúde (projeto de pesquisa). São Paulo, Fundação SEADE, 1990.

20. FLEISS, J. L. Statistical methods for rates and proportions. New York, John Wiley and Sons, 1981. p. 212-36.

21. FUNDAÇÃO SEADE. A mortalidade infantil em São Paulo no $1^{\circ}$ semestre de 1990. Conjunt. Demogr., 33-51, 1991.

22. GOULET, L. Maternal and child care. Inf. Dép. Soc. I'Univ. Montréal, 5 (6) 1985.

23. GUIMARÃES, J. J. L. \& FISCHMANN, A. Infant mortality among chanty town residents in the municipality of Porto Alegre, RS, Brazil. Bull. Panam. Health Organ., 19: 235-51, 1985.

24. HARTZ, Z. M. A.; CHAMPAGNE, F.; CONTANDRIOPOULOS, A. P.; DENIS, J. L. L'analyse d'implantation du Programme de Santé Maternelle et Infantile (PMI) dans deux Systèmes Locaux de Santé (SYLOS) au "Nordeste" du Brésil (mimeografado).

25. JOUGLA, E.; DUCIMETIERE, P.; BOUVIER-COLLE, M. H., HATTON, F. Rélation entre le niveau de développement du système de soins et le niveau de mortalité évitable selon les départements français. Rév. d'Épidemiol. Santé Publique, 35: 365-77, 1987.

26. KLEINMAN, J. C. Infant mortality. Statistical Notes for Health Planners, Vol. 2. Washington: NCHS, 1977.

27. LOPEZ, A. D. Décès évitables: vue d'ensemblé. Rapp. Trim. Statist. Sanit. Mond., 42: 2-3, 1989.

28. MONTEIRO, M. F. G. Considerações sobre os fatores sócioeconômicos e diferenciais de mortalidade infantil nas regiões metropolitanas do Brasil, calculados através do método de caso-controle. In: Perfil estatístico de crianças e mães no Brasil. Brasília, UNICEF/IBGE, 1986. p. 78-92.

29. MURNAGHAN, J. H. Heath indicators and information systems for the year 2000. Ann. Rev. Public. Health, 2: 299$361,1981$.

30. NAKAMURA, R. M.; KING, R.; KIMBALL, E. H.; OYE, R. K.; HELGERSON, S. D. Excess infant mortality in an American Indian Population, 1940-1990. JAMA, 266: 22448, 1991.

31. NERSESIAN, W. S. Infant mortality in socially vulnerable populations. Ann. Rev. Public Health, 9: 361-77, 1988.

32. PAIM, J. L.; COSTA, M. C. N.; CABRAL, V.; MOTA, I. A.; NEVES, R. B. B. Spatial distribution of infant mortality an certain socioeconomic variables in Salvador, Bahia, Brasil. Bull. PAHO, 21; 225-39, 1987.

33. PHAROAH, P. O \& MORIIS, J. N. Postneonatal mortality. Epidemiol. Rev., 1: 170-83, 1979.

34. PLAUT, R. \& ROBERTS, E. Preventable mortality: indicator or target? Applications in developing countries. World Health Stat. Q., 42: 4-15, 1989. 
35. POTVIN, L. \& CHAMPAGNE, F. Utilization of administrative files in health research. Soc. Indicators Res., 18: 409-23, 1986.

36. POTVIN, L. L'utilisation des fichiers admistratifs pour des fins de recherche en santé. Montréal, Université de Montréal, 1984.

37. PUFFER, R. R. New approaches for epidemiologic studies of mortality statistics. Bull. PAHO, 23: 365-83, 1989.

38. PUFFER, R. R. \& SERRANO, C. V. Pattems of mortality in childhood. Washington, PAHO, 1973. (PAHO - Scientific Publication 262).

39. ROSEMBERG, H. M. The nature and accuracy of case-ofdeath data. In: Report of the Workshop on Improving Causeof-Death Statistics. Washington, D. C., NCHS, 1989.

40. ROSEMBERG, H. M. Mechanisms by which cause-of-death data get to the policy maker. In: Report of the Workshop on Improving Cause-of Death Statistics. Washington, D.C., NCHS, 1989.

41. RUTSTEIN, D. D.; BERENBERG, W.; CHALMERS, T. C.; CHILD, C. G.; FISHMAN, A. P.; PERRIN, E. B. Measuring the quality of medical care: a clinical method. N. Engl. J. Med., 294: 582-8, 1976.

42. RUTSTEIN, D. D. Monitoring progress and failure: sentinel health events (unnecessary diseases, disabilities an untimely deaths). In: Measurement in health promotion and protection. Copenhagen, WHO/IEA, 1987. p. 95-212.

43. SAAD, P. M. Mortalidade infantil por causas no Estado de São Paulo (Brasil) em 1983. Rev. Saúde Pública, 20: 481-8, 1986.

44. SAWYER, D.; FERNANDES-CASTILLA, R.; MONTEMOR, R. L. M. The impact of urbanization and industrialization on mortality in Brazil. World Health Stat. Q., 4: 8497, 1987.

45. SIMÕES, C. C. S. \& ORTIZ, L. P. A mortalidade infantil no Brasil nos anos 80. In: Crise e infância no Brasil: o impacto das políticas de ajustamento econômico. São Paulo, IPE/ USP, 1988. p. 243-68.

46. SIMÕES, C. Novas estimativas de mortalidade infantil. In: Fundação IBGE. Mortalidade infantil e saúde na década de 80. Rio de Janeiro, FIBGE/UNICEF, 1989.

47. STREINER, D. L. \& NORMAN, G. R. Health measurement scales. New York, Oxford University Press, 1989.

48. SZWARCWALD, C. L.; CHEQUER, P.; CASTILHO, E. A. Tendências da mortalidade infantil no Brasil nos anos 80 . Inf. Epidemiol. SUS, 1: 35-50, 1992.

49. SZWARCWALD, C. L.; LEAL, M. C.; JOURDAN, A. M. F. Mortalidade infantil: o custo social do desenvolvimento brasileiro. In: Leal, M. C.; Sabroza, P. C.; Rodriguez, R. H.; Buss, P. M., org. Saúde, ambiente e desenvolvimento. São Paulo, Hucitec Abrasco, 1992. v. 2, p. 251-78,

50. UNICEF/GOVERNO DO RIO GRANDE DO NORTE. $A$ situação da criança no Rio Grande do Norte. Natal, 1990.

51. UNICEF. Brazil's social debt to mothers and children: what can be done until year 2000? Brasília, 1986.

52. UNICEF. The state of the world children 1991. New York, Oxford Univ. Press, 1991.

53. WALLACE, H. M. Health care of children in developing countries. In: Wallace, H. M. \& Giri, K. ed. Health care of women and children in developing countries. Oaklar, CA, Third Publ. Company, 1990.

54. WASH, J. \& WAREEN, K. Selective primary health care: an interim strategy for disease control in developing countries. Soc. Sci. Med., 14C: 145-63, 1980.

55. YUNES, J. \& CAMPOS, O. Health services in the metropolitan region of São Paulo. Bull. PAHO, 23: 350-6, 1989.

56. ZAHNISER, S. C.; KENFRICK, J. S.; FRANKS, A. L.; SAFTLAS, A. F. Trends in obstetric operative procedures, 1980 to 1987. Am. J. Public. Health, 82: 1340-4, 1992.

\section{ANEXO}

\section{CORRESPONDÊNCIA ENTRE OS NÍVEIS DE TAXA DE MORTALIDADE INFANTIL (TMI), O ÍNDICE DE MORTALIDADE INFANTIL (IMI) E O ÍNDICE DE MORTES EVITÁVEIS (PDI)}

\begin{tabular}{lccl}
\hline TMI & IMI & PDI & Classificação de Bourgeois-Pichat \\
\hline 0,300 & 100 & 70 & Rocha macia \\
0,215 & 90 & 60 & (grande percentagem de óbitos de origem infecciosa) \\
0,150 & 80 & 50 & \\
0,100 & 70 & 40 & \\
\hline 0,070 & 60 & 30 & Rocha intermediária \\
0,045 & 50 & 20 & (novos declínios possíveis através do desenvolvimento \\
0,030 & 40 & 10 & social) \\
\hline 0,020 & 30 & 0 & Rocha dura \\
0,010 & 20 & & (grande percentagem de óbitos devido a anomalias \\
0,008 & 10 & & congênitas e a certas causas perinatais) \\
0,005 & 0 & & \\
\hline
\end{tabular}

